\title{
Determinação de Espectros de Energia de Elétrons Clínicos a partir de Curvas de Porcentagem de Dose em Profundidade (PDP) utilizando o Método de Recozimento Simulado Clássico Determination of Electron Clinical Spectra from Percentage Depth Dose (PDD) Curves by Classical Simulated Annealing Method
}

\author{
Jorge. H. Wilches Visbal ${ }^{1}$, Alessandro M. Costa ${ }^{2}$ \\ ${ }^{1}$ Faculdade de Filosofia, Ciências e Letras de Ribeirão Preto (USP), Ribeirão Preto (SP), Brasil \\ ${ }^{2}$ Faculdade de Filosofia, Ciências e Letras de Ribeirão Preto (USP), Ribeirão Preto (SP), Brasil
}

\begin{abstract}
Resumo
As curvas de porcentagem de dose em profundidade representam um conjunto importante de dados para feixes de elétrons pois descrevem claramente as propriedades dosimétricas destes. Usando uma teoria de transporte acurada ou o método Monte Carlo encontram-se diferenças obvias entre a PDP de feixes de elétrons monoenergéticos e a de feixes de elétrons clínicos à energia nominal do acelerador em um objeto simulador de água. Em radioterapia, o espectro de energia de elétrons deve ser considerado para aprimorar a acurácia do cálculo da dose toda vez que o feixe de elétrons que atinge a superfície do objeto simulador de água após atravessar às estruturas do acelerador e o ar, não é mais monoenergetico. Existem três abordagens principais para extrair o espectro de energia de elétrons desde curvas de PDP: Método Monte Carlo, Medição Direta e Reconstrução Inversa. Neste trabalho será apresentado o método de Recozimento Simulado Clássico como uma abordagem prática, consistente e simples de reconstrução inversa como sendo uma boa alternativa aos outros dois métodos.
\end{abstract}

Palavras-chave: recozimento simulado; espectros de energia; elétrons; radioterapia; porcentagem de dose em profundidade; Matlab; otimização; PENELOPE.

\begin{abstract}
Percentage depth dose of electron beams represents an important item of data in radiation therapy treatment since it describes the dosimetric properties of these. Using an accurate transport theory, or the Monte Carlo method, has been shown obvious differences between the dose distribution of electron beams of a clinical accelerator in a water simulator object and the dose distribution of monoenergetic electrons of nominal energy of the clinical accelerator in water. In radiotherapy, the energy spectrum of electrons should be considered to improve the accuracy of dose calculation, because the electron beams that reach the surface traveling through internal structures of accelerator are not in fact monoenergetic. There are three principal approaches to obtain electron energy spectra from central PDP: Monte Carlo Method, Direct Measurement and Inverse Reconstruction. In this work, it will be presented the Simulated Annealing method as a practical, reliable and simple approach of inverse reconstruction as being an optimal alternative to other options.
\end{abstract}

Keywords: simulated annealing; electrons; energy spectra; radiation therapy; percentage depth dose; Matlab; optimization, PENELOPE.

\section{Introdução}

Experimentos têm mostrado diferenças obvias entre as curvas de porcentagem de dose em profundidade de feixes de elétrons de aceleradores clínicos e as curvas de porcentagem de dose em profundidade de feixes de elétrons monoenergéticos à energia nominal do acelerador em um objeto simulador de água1.

Essas diferenças são devidas ao fato dos elétrons clínicos que atingem a superfície do objeto simulador não serem realmente monoenergéticos.

No percurso da fonte de radiação até a superfície do objeto simulador, os elétrons clínicos interagem tanto com as estruturas internas do cabeçote do acelerador (folhas espalhadoras, colimadores e aplicador) quanto com o $\mathrm{ar}^{2}$.

Nestas interações, elétrons de baixa energia são gerados e injetados nas primeiras camadas de água dado seu curto alcance. Entretanto, fótons de alta energia também são produzidos, mas depositam sua energia nas profundezas do objeto simulador.

Em consequência, a dose efetivamente depositada no eixo central difere notavelmente daquela produzida em um hipotético feixe monoenergetico à energia nominal, particularmente, perto da superfi$\mathrm{cie}^{1,2}$.

Resulta evidente que, para se conseguir um cálculo acurado e preciso da porcentagem de dose em profundidade de qualquer feixe de elétrons, o espectro de energia incidente tem de ser levado em consideração $0^{1,3}$.

Pois bem, o problema a ser resolvido é: "como determinar o espectro de energia de elétrons clínicos a partir da curva de PDP do eixo central".

Existem pelo menos três abordagens para resolver este problema ${ }^{3,4}$ : 
1. Simulação Monte Carlo: consiste no modelamento da fonte por meio do Monte Carlo.

Embora ser o método mais acurado ${ }^{4}$, é só um método de simulação com pouca flexibilidade que precisa construir um modelo complexo ajustado a um tipo particular de acelerador.

Aliás, requer perícia e consome muito tempo para gerar um conjunto completo e útil de dados de phase-space para uso clínico ${ }^{5}$.

2. Medição Direta: consiste na determinação experimental do espectro através de equipamentos como: espectroscópio de elétrons ${ }^{1}$, espectrômetro de espalhamento Compton ${ }^{3,4}$ e espectrômetro magnético de elétrons ${ }^{5,6}$.

A despeito de ser um método acurado, consome muito tempo, é perturbador e proibitivamente custoso.

3. Reconstrução Inversa: consiste na determinação do espectro a partir de um modelamento matemático. Este método resolve o problema inverso: obter o espectro a partir de dados de $P D P^{1,3,4}$, dispondo de ferramentas de otimização sejam elas estocásticas ou deterministas. No entanto não ser o padrão ouro, o fato de ser simples, rápido e relativamente acurado, tornao como a melhor abordagem ${ }^{3}$, dentre as outras.

Algoritmos úteis para estabelecer a contribuição na dose dos fótons de freamento gerados no cabeçote do acelerador têm sido investigados ${ }^{2,7,8}$. A influência do espalhamento angular inicial no espectro de energia dos elétrons incidentes também tem sido reportada ${ }^{2,9}$.

Neste artigo nos limitaremos a reconstruir o espectro de elétrons ignorando tanto a contribuição dos fótons contaminantes de alta energia quanto a contribuição do espalhamento angular. Em outras palavras, reconstruiremos $0<<$ som puro $>>$ dos elétrons que atingem a superfície do objeto simulador de água.

\subsection{Modelamento do Problema}

Suponha que o feixe de elétrons tem um espectro de energias $\varphi(E)$ antes de atingir a superfície do objeto simulador. $D(E, z)$ é a distribuição de dose gerada por elétrons monoenergéticos de Energia $E$ em água e $D(z)$ é a distribuição de dose medida/simulada no eixo central do feixe.

Por meio da Equação-Integral de Fredholm de primeiro tipo é possível enlaçar o espectro reconstruído com a PDP medida/simulada ${ }^{3,4,10}$ aproveitando um conjunto de curvas de PDP monoergeticas que agem como kernel dentro da integral.

$$
D(z)=\int_{0}^{E_{\max }} D(E, z) \varphi(E) d E
$$

Discretizando, através de algum método numérico, a equação-integral pode ser expressa assim:

$$
D(z)=\sum_{i}^{n} D\left(E_{i}, z_{j}\right) \varphi\left(E_{i}\right) \Delta E=K \varphi
$$

Onde $K=\left[D\left(E_{i}, z_{j}\right) \Delta E\right]$ é chamada de função ker$n e l, n$ é o número máximo de feixes monoenergéticos usados para construir a função kernel e $D(z)$ é a PDP gerada pelo feixe clínico/polienergético simulado.

A equação (3) é geralmente um sistema linear malcolocado ${ }^{1,10,11}$ daqui que métodos comuns de resolução de sistemas lineares tais como método de matriz inversa $(m=n)$ ou método de matriz inversa generalizada $(m \neq n)$ não costumam ser satisfatórios ${ }^{1}$.

Vários métodos ${ }^{3-7,10}$ têm sido desenvolvidos a fim de extrair o espectro de elétrons da curva de PDP do eixo central, fazendo uso da equação (1), a causa de ser este um problema mal-posto ${ }^{1,310}$.

\subsection{Método de Recozimento Simulado Clássico}

Recozimento Simulado é um método metaheurístico proposto por Scoot Kirkpatric et $\mathrm{al}^{12}$ que simula o processo de recozimento de metais.

Recozimento é o processo utilizado para fundir um metal, onde este é aquecido a uma temperatura elevada e em seguida é resfriado lentamente, a fim de que o produto final seja uma massa homogênea ou estado cristalino livre de imperfeições ${ }^{13-15}$.

Durante o recozimento o material passa por vários estados possíveis tal que um resfriamento rápido conduz a produtos metaestáveis de maior energia interna (relacionado com mínimos locais) enquanto um resfriamento lento conduz a produtos mais estáveis de estrutura consistente, de menor energia (relacionado com o mínimo global) ${ }^{14}$.

O método é idôneo em problemas de otimização de limites não-constrangidos/constrangidos e de grande escala, onde o mínimo global poderia estar encoberto entre muitos mínimos locais ${ }^{5}$.

Também destaca nos casos em que a função objetivo a ser minimizada ou maximizada é discreta e/ou muito comprida 5 .

Diferentemente de alguns métodos deterministas que convergem rapidamente aos pontos nas proximidades associados a mínimos locais ${ }^{5}$ (estados metaestáveis), Recozimento Simulado é capaz de "pular" esses extremos locais e aos poucos atingir o mínimo global ou estado estável, i.e., de mínima energia possível $\left.\right|^{5,14}$. 


\section{Materiais e Métodos}

Para a obtenção dos resultados utilizou-se a função sim_anl() desenvolvida por Hector Corte $^{16}$ e baseada no Método Clássico de Simulated Annealing ${ }^{12}$.

A função objetivo a ser minimizada foi a de Regularização de Tikhonov Clássica ${ }^{17} \mathrm{com}$ fator de regularização perto do ótimo dado pela curva $L$ e matriz de regularização de ordem 1 , para todas as três energias analisadas.

O Kernel, foi construído a partir de curvas de PDP monoenergéticas simuladas em PENELOPE-MC, com distância fonte-superfície de $100 \mathrm{~cm}$ e tamanho de campo $10 \times 10 \mathrm{~cm}^{2}$.

O vetor de termos independentes constitui a PDP simulada (para as 3 energias em questão) em PENELOPE a partir dos espectros de energia de um Acelerador Clinac $2100 \mathrm{C}^{18}$. Para a digitalização do espectro usou-se o software Engauger Digitizer 4.1.

Os valores da energia mais provável bem como 0 da energia máxima foram extraídos da PDP simulada por meio de relações bem conhecidas ${ }^{7}$.

A tentativa inicial de solução, um vetor com a seguinte distribuição: o bin correspondente à energia mais provável foi assignado o valor 1 , aos bin adjacentes o valor de 0.5 , por sua vez, aos adjacentes a estes 0.25 , e a estes 0.125 . O resto dos bin 0.01 , exceto $\mathrm{o}$ associado à energia de corte $\mathrm{o}$ qual tem valor de 0 .

O delta de energia foi de $125 \mathrm{keV}$ para o espectro de $6 \mathrm{MeV}$ e de $9 \mathrm{MeV}$ enquanto que de $250 \mathrm{keV}$ para o de $12 \mathrm{MeV}$ de energia nominal.

Resumidamente o código fonte trabalha do seguinte jeito ${ }^{13,16}$ :

- Estabelece-se uma tentativa inicial da solução, as restrições superior e inferior das componentes, o número máximo de iterações e a tolerância da função.

- A tentativa inicial coloca-se como sendo a primeira solução do problema. A função objetivo é avaliada na tentativa inicial.

- Para cada iteração gera-se, aletoriamente, um passo que é adicionado à tentativa inicial para formar o ponto de teste. Repete-se isto 500 vezes para estimular o alcance do mínimo global.

- Sempre que a função objetivo no ponto de teste for menor que o inicial, passa a ser o novo ponto inicial. No entanto, há uma certa probabilidade de um ponto de teste que aumentar 0 valor da função de custo seja aceito. A ideia nisto é evitar ficar preso em extemos locais.

- Repete-se o ciclo $\mathrm{n}$ vezes até o número máximo de iterações, com o intuito de garantir a consecução do mínimo global. O número total de iterações para convergência foi de um miIhão ( $\mathrm{n}=2000$ iterações para cada ciclo de 500 iterações: $2000 \times 500=1$ e6 iterações totais).

O software utilizado na programação: Matlab R2015a, plataforma Win 7, SP1. CPU: $2.3 \mathrm{GHz}$ e RAM: $4 \mathrm{~Gb}$.

\section{Resultados}

A validação do método foi feita por comparação entre as PDP original e reconstruída. Ambas foram obtidas por simulação Monte Carlo usando o código PENELOPE.

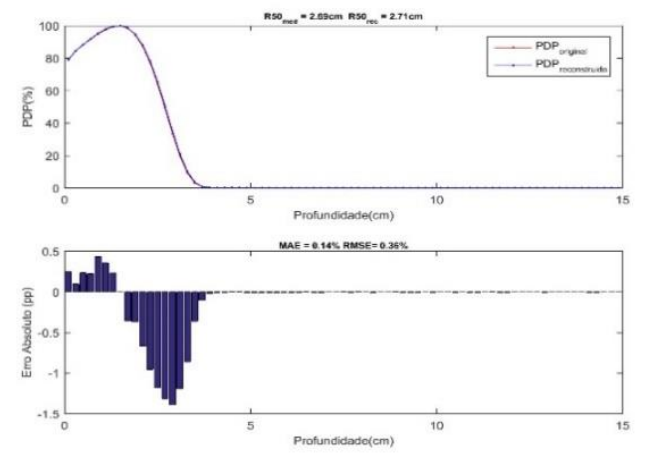

Figura 1. Comparação entre PDP do espectro original e a do espectro reconstruído. Energia nominal $6 \mathrm{MeV}$.

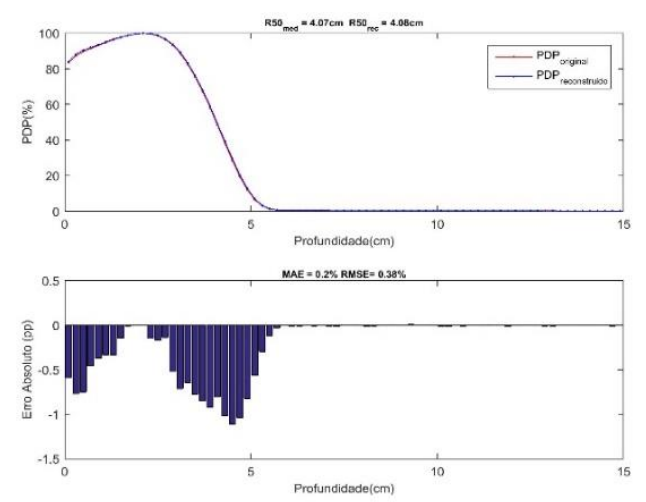

Figura 2. Comparação entre PDP do espectro original e a do espectro reconstruído. Energia nominal $9 \mathrm{MeV}$.

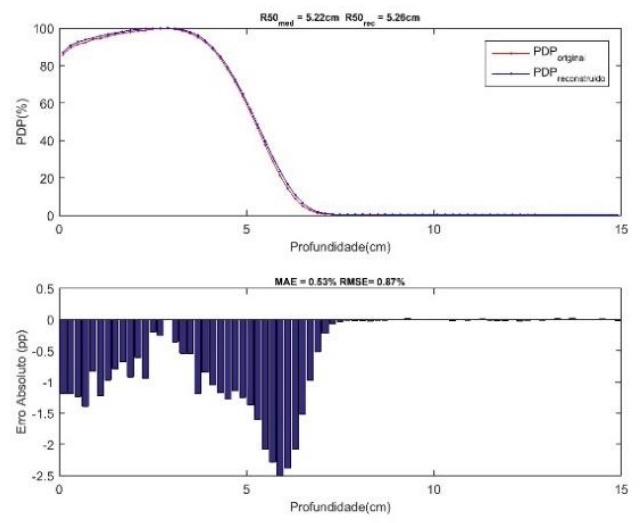

Figura 3. Comparação entre PDP do espectro original e a do espectro reconstruído. Energia nominal $12 \mathrm{MeV}$.

Nas figuras, MAPE: Erro Médio Percentual Absoluto ${ }^{19}$, RMSE: Erro RMS ${ }^{19}$ e pp: pontos porcentuais. 


\section{Discussão}

Para todas as energias, o MAPE e o RMSE ficaram abaixo do $1 \%$.

Atendendo aos padrões típicos de aceitação clínica segundo o Índice Gamma (2-5\%/2-5mm) ${ }^{20,21}$ a reconstrução espectral obtida pelo método de recozimento simulado clássico encontra-se na faixa de aceitação toda vez que em nenhum dos espectros a distância entre os pontos de interesse jamais superou os $2 \mathrm{~mm}$ e entre as PDP nunca foi maior do que $3 \%$ como visto nas figuras 1,2 e 3 .

$\mathrm{O}$ valor-p para energia de $6 \mathrm{MeV}$ correspondeu a 0.98 , para $9 \mathrm{MeV}, 0.97$ e para $12 \mathrm{MeV}, 0.93$, indicando não existência de diferença estatisticamente significativa entre as PDP para cada energia.

\section{Conclusões}

O método de Recozimento Simulado Clássico é capaz de reconstruir espectros dentro dos limites de aceitação clínica.

O uso da regularização de Tikhonov e a curva $L$ garante uma escolha apropriada do fator de regularização que conduz a um resultado próximo ao original.

O valor do fator de regularização, não sempre dá resultados esperados pelo que sua escolha é crítica.

Diminuir o delta de energia para feixes com maior energia nominal poderia melhorar os resultados.

\section{Agradecimentos}

Os autores agradecem o apoio da Comissão de Aperfeiçoamento de Pessoal do Nível Superior (CAPES) e da Fundação de Amparo e Pesquisa do Estado de São Paulo (FAPESP).

\section{Referências}

1. Luo $Z$ and Jette D. On the possibility of determining an effective energy spectrum of clinical electron beams from percentage depth dose (PDD) data of broad beams. Physics in Medicine and Biology 44 N177-N182. 1999.

2. Brahme, A., and H. Svensson. "Radiation beam characteristics of a $22 \mathrm{MeV}$ microtron." Acta radiologica: oncology, radiation, physics, biology 18.3: 244-272. 1979

3. Gui Li et al, in: Realization and Comparison of Several Regression Algorithms for Electron Energy Spectrum Reconstruction. Chin.Phys.Lett. Vol 25, No.7, 2710. 2008.

4. Gui Li et al, in: Electron spectrum reconstruction as nonlinear programming model using micro-adjusting algorithm. APCMBE 2008, IFMBE Proccedings 19, pp. 451-454, 2008.

5. Carletti C, Meoli P and Cravero W. A modified simulated an nealing algorithm for parameter determination for a hybrid virtual model. Phys. Med.Biol 51 3941-3952. 2006.

6. Faddegon B and Blevis I. Electron spectra derived from depth dose distribution. Medical Physics 27, 514-526.2000.

7. Deng J, Jiang S, Pawlicki J and Ma C. Derivation of electron and photon energy spectra from electron beam central axis depth dose curves. Physics in Medicine and Biology 46 , 1429-1449. 2001

8. Klevenhagen, S. C. "An algorithm to include the bremsstrahlung contamination in the determination of the absorbed dose in electron beams." Physics in Medicine and Biology 39.7, 1103. 1994.

9. Chvetsov A and Sandison G. Angular correction in reconstruction of electron spectra from depth dose distributions. Medical Physics 30, 2155-2158. 2003.

10. Chvetsov A and Sandison G, in: Reconstruction of electron spectra using singular component decomposition. Medical Physics 29, 578-591. 2002.
11. Luo Z. A numerical method for solving the Fredholm integral equation of the first kind and its application to restore the folded radiation spectrum. Nucl. Instrum. Methods A 255152. 1987.

12. Kirkpatric $S$ et al. Optimization by Simulated Annealing. Science New Series Vol 220 No 4598. p. 671-680. 1983.

13. Won Y. Yang, Wenwu Cao, Tae-Sang Chung, John Morris. Applied Numerical Methods Using MATLAB. John Whiley \& Sons, 2005.

14. Lorena LA. Otimização Combinatória, CAP 254. Laboratório Associado de Computação e Matemática Aplicada, INPE, Brasil. 2007.

15. Haeser G e Gomez M. Aspectos teóricos de Simulated Annealing e um algoritmo duas fases em otimização global. Mat. Apl. Comput., 9, No. 3, 395-404. 2008.

16. Mathworks.com [homepage on the Internet]. Oviedo, Espanha: Simulated Annealing Optimization [cited/acesso U2016/Abr/7]. Disponível em http://www.mathworks.com/ matlabcentral/fileexchange/33109-simulated-annealing-optimization/content/sim anl.m.

17. Per Christian Hansen. Regularization Tools: A Matlab Package for Analysis and Solution of Discrete III-Posed Problems. Numerical Algorithms 6, 1-35. 1994.

18. Rogers D, Faddegon B, Ding G, Ma C-M, We J and Mackie T. BEAM: a Monte Carlo code to simulate radiotherapy treatment units. Medical Physics 22 503-24. 1995.

19. Chai, Tianfeng, and Roland R. Draxler. "Root mean square error (RMSE) or mean absolute error (MAE)? -Arguments against avoiding RMSE in the literature." Geoscientific Model Development 7.3: 1247-1250. 2014.

20. Faddegon, B. A., and I. Blevis. "Electron spectra derived from depth dose distributions." Medical Physics 27.3: 514-526. 2000

21. Low, Daniel A., et al. A technique for the quantitative evaluation of dose distributions. Medical Physics 25.5: 656-661. 1998

\section{Contato:}

Jorge $\mathrm{H}$. Wilches Visbal.

Sala 411, Bloco 5, Departamento de Física da Universidade de São Paulo. Av. Bandeirantes, 3.900 Monte Alegre, Cidade Universitária de Ribeirão Preto. CEP: 14040-900. Ribeirão Preto, SP, Brasil. E-mail: jhwilchev@usp.br. 